\title{
Nanofat 2.0: Experimental Evidence for a Fat Grafting Rich in Mesenchymal Stem Cells
}

\author{
D. LO FURNO ${ }^{* *}$, S. TAMBURINO ${ }^{2 *}$, G. MANNINO ${ }^{1}$, E. GILI ${ }^{3}$, G. LOMBARDO ${ }^{2}$, \\ M. S. TARICO ${ }^{2}$, C. VANCHERI ${ }^{3}$, R. GIUFFRIDA ${ }^{1}$, R. E. PERROTTA ${ }^{2}$ \\ ${ }^{*}$ These authors contributed equally to this work.
}

${ }^{1}$ Department of Biomedical and Biotechnological Sciences, University of Catania, Catania, Italy, ${ }^{2}$ Department of Plastic and Reconstructive Surgery, Cannizzaro Hospital, University of Catania, Italy, ${ }^{3}$ Department of Clinical and Experimental Medicine, University of Catania, Catania, Italy

Received July 26, 2016

Accepted December 5, 2016

On-line April 12, 2017

\section{Summary}

Different strategies have been developed in the last decade to obtain fat grafts as rich as possible of mesenchymal stem cells, so exploiting their regenerative potential. Recently, a new kind of fat grafting, called "nanofat", has been obtained after several steps of fat emulsification and filtration. The final liquid suspension, virtually devoid of mature adipocytes, would improve tissue repair because of the presence of adipose mesenchymal stem cells (ASCs). However, since it is probable that many ASCs may be lost in the numerous phases of this procedure, we describe here a novel version of fat grafting, which we call "nanofat 2.0", likely richer in ASCS, obtained avoiding the final phases of the nanofat protocol. The viability, the density and proliferation rate of ASCs in nanofat 2.0 sample were compared with samples of nanofat and simple lipoaspirate. Although the density of ASCs was initially higher in lipoaspirate sample, the higher proliferation rate of cells in nanofat 2.0 virtually filled the gap within 8 days. By contrast, the density of ASCs in nanofat sample was the poorest at any time. Results show that nanofat 2.0 emulsion is considerably rich in stem cells, featuring a marked proliferation capability.

\section{Key words}

Fat grafting - Adipose stem cells - Cell proliferation • Regenerative medicine

\section{Corresponding author}

R. Giuffrida, Department of Biomedical and Biotechnological Sciences, University of Catania, Catania, Italy. E-mail: giuffros@unict.it

\section{Introduction}

Regenerative properties and multilineage differentiation ability (Calabrese et al. 2015, Lo Furno et al. 2013, Ullah et al. 2015) of mesenchymal stem cells (MSC) have been exploited in the last decade to improve fat grafting techniques. In fact, by early methods of adipose tissue transplantation, significant portions of the transplanted tissue were often reabsorbed, perhaps for a poor blood supply due to insufficient neoangiogenesis. Adipose derived MSCs (ASCs) and their secretomes seem able to induce production of the extracellular matrix, new collagen deposition and early revascularization (Butala et al. 2012, Kalinina et al. 2015, Charles-de-Sá et al. 2015).

Various techniques have been designed to obtain a fat graft as rich as possible of ASCs. A method defined Cell-Assisted Lipotransfer (CAL) was developed in mice in 2006 (Matsumoto et al. 2006). Before reinjection, the standard lipoaspirate was supplemented with freshly prepared stromal vascular fraction (SVF), the portion of centrifuged adipose tissue containing ASCs. By this method, satisfactory results were also observed in human trials (Yoshimura et al. 2008), considering that significant breast volume augmentation was obtained in most patients. A two-step procedure was introduced by Kølle et al. (2013). The fat graft from fresh lipoaspirate was enriched with previously expanded ASCs, obtained from a 14 days earlier liposuction. Bianchi et al. (2013) 
described a method that avoids the use of collagenase, or previous cell cultures in vitro. Following sequential filtering steps of lipoaspirate they obtained small adipose tissue clusters (0.2-0.8 mm), termed "lipogems", which could be used for reinjections in the same surgical setting. A similar strategy, called "nanofat" grafting, has been proposed by Tonnard et al. (2013) for skin rejuvenation, especially to treat particular areas, such as eyelids, lips or fine rhytides. In that study, characteristics of nanofat were compared with other fat grafts such as "macrofat" and "microfat". Macrofat consists of lipoaspirate harvested through a cannula with large side holes ( $2 \times 7 \mathrm{~mm})$; microfat is obtained through a cannula with smaller side holes ( $1 \mathrm{~mm}$ in diameter); derived from microfat, nanofat is obtained after several steps of emulsification and filtration. This liquid suspension can be injected in a more superficial plane through finer needles (27 gauge). Indeed, the authors describe appreciable clinical results without major unwanted side effects such as infections, fat cysts or granulomas.

The aim of the present study was to obtain a fat graft even richer in ASCs. Supposing that a considerable amount of ASCs may be lost in the several steps of Tonnard's procedure, we designed a more simplified method, by skipping the final two phases of nanofat protocol: the last filtration and the final squeezing of the fat suspension through sterile gauze. In this way, a larger amount of ASCs should be preserved in this minimally manipulated final emulsion, which we called "nanofat 2.0", to be utilized for therapeutic applications in plastic and reconstructive surgery. To verify this hypothesis, we expanded ASCs obtained from three samples: simple microfat lipoaspirate, nanofat emulsion according to Tonnard et al. (2013), nanofat 2.0 emulsion, prepared by following our modifications. For each sample, we investigated the density of stem cells, their viability and proliferation rate, as well as their immunophenotype. To this aim, immunocytochemistry, flow cytometry techniques and MTT assay were utilized.

\section{Methods}

\section{Fat grafting preparation}

A total of 8 patients, from 30 to 60 years old (mean age 40), were included in the present investigation, after signing an informed consent to use the lipoaspirate for experimental procedures. The study was performed following the standards of the local ethics committee and in accordance to Declaration of Helsinki (2000). Fat tissue was harvested from the abdominal region through a multiport 3-mm cannula with small sharp side holes ( $1 \mathrm{~mm}$ in diameter) under low negative pressure through a hand-held syringe aspiration, during standard procedures of lipofilling or abdominoplasty. From each subject, a portion of standard lipoaspirate was kept apart in a sterile recipient as a microfat sample, while the remaining portion was used to prepare in parallel the nanofat and nanofat 2.0 emulsions. Nanofat emulsion was made following rigorously the protocol of Tonnard et al. (2013). In this case, the lipoaspirate was rinsed, filtered and mechanically emulsified by shifting it between two 10 -cc syringes connected to each other by a Luer Lock connector. After 30 passages, the fatty emulsion was filtered again over the sterile nylon cloth and the effluent nanofat was collected in a second sterile recipient. The third sample, the nanofat 2.0, was obtained through the same procedure described above, but avoiding the final phases of filtering and squeezing the fat emulsion through the gauze. These three samples were then processed for comparative analysis.

\section{Oil red $O$ staining}

Soon after the harvest, microscopic preparations from each sample were obtained by spreading the lipofilling material on a glass slide, as uniformly as possible. After fixation to flame, each sample was washed with isopropanol $60 \%$ and covered with Oil red $\mathrm{O}$ working solution for $60 \mathrm{~min}$. After a final rinse with distilled water, slides were observed at the optical microscope. Digital images were acquired using a Leica DMRB equipped with a computer assisted Nikon digital camera.

\section{Cell growth}

In order to isolate and expand ASCs, cell cultures from each of the three different samples (microfat, nanofat and nanofat 2.0) were carried out following a protocol reported by Zuk et al. (2002). The same volume of each sample (up to $10 \mathrm{ml}$ ) was incubated for $3 \mathrm{~h}$ at $37^{\circ} \mathrm{C}$ with an equal volume of serum-free DMEM-low glucose (DMEM-1g) containing $0.075 \%$ of type I collagenase. After blocking collagenase activity by an equal volume of DMEM-1g containing $10 \%$ of Fetal Bovine Serum (FBS), samples were centrifuged at $1200 \mathrm{rpm}$ for $10 \mathrm{~min}$. Pellets obtained were resuspended in PBS, filtered through a $100 \mu \mathrm{m}$ nylon cell strainer and again centrifuged. These final pellets were resuspended with DMEM-1g (10\% FBS, pen/strep $1 \%)$ containing 
$1 \%$ of Mesenchymal Stem Cells Growth Medium (MSCGS). Finally, cells were plated in T75 culture flasks and incubated at $37{ }^{\circ} \mathrm{C}$ with $5 \% \mathrm{CO}_{2}$. After seeding, cell cultures were continuously monitored to evaluate the presence and growth of adherent cells. In each culture, the cellular density was indexed by averaging the number of cells found in 5 microscopic fields at magnification of $10 x$, in zones of higher cell density. Their growth rate was calculated at $24 \mathrm{~h}, 4$ days and 8 days, by the following formula:

(final number - initial number) / initial number

At 8-10 days, confluence was usually reached in microfat and nanofat 2.0 samples, while cell population in nanofat sample was quite modest. However, in order to guarantee the same time course for all samples, cultures were trypsinized, resuspended and seeded for the second passage. To obtain in each sample a congruous number of cells to investigate, an enriching strategy was adopted between passages: the entire population of nanofat sample was seeded, but only one third of the other two samples was used. After 5-6 days, cells often reached $80 \%$ of confluence in microfat and nanofat 2.0 cultures, although in nanofat culture they hardly reached $50 \%$. After further 5-6 days in the third passage, since the number of cells was quite satisfactory in all cultures, they were processed for MTT assay and to determine their cytochemical features.

\section{Determination of ASCs markers}

To verify the MSC nature of cultured cells, immunocytochemistry and flow cytometry was carried out to identify in each sample the presence of stem cell surface markers. For immunocytochemistry, cells were sub-cultured in a 96-well culture dish for 2 days. They were then washed with PBS, fixed with $4 \%$ paraformaldehyde in PBS for $30 \mathrm{~min}$, and left for $30 \mathrm{~min}$ in a $5 \%$ solution of normal goat serum. Finally, cells were incubated overnight at $4{ }^{\circ} \mathrm{C}$ with primary antibodies: CD44 (1:200; Abcam, Boston, MA, USA), CD90 (1:100; Abcam), CD105 (1:100; Abcam), CD14 (1:200; Abcam), CD34 (1:200; Novus Biologicals, Littleton, CO, USA), CD45 (1:200; Abcam). The following day, cells were washed with PBS and incubated for $1 \mathrm{~h}$ at room temperature with FITC-conjugated goat anti-mouse or FITC-conjugated goat anti-rabbit secondary antibodies (Abcam). As a control, in each sample, the specificity of immunostaining was verified by omitting incubation with the primary or secondary antibodies. As a rule, cell nuclei were counterstained with DAPI for $10 \mathrm{~min}$, to verify the presence of cells, immunopositive or not. The excitation wavelength was $488 \mathrm{~nm}$ for FITC and $350 \mathrm{~nm}$ for DAPI. Immunostaining was evaluated taking into account the signal-to-noise ratio of immunofluorescence. Digital images were acquired using a Leica inverted fluorescence microscope equipped with a computer assisted Nikon digital camera.

For flow cytometry, cells were sub-cultured in a 6-well culture dish for 2 days. Cells were then washed with PBS, again trypsinized, and fixed with $2 \%$ paraformaldehyde for $20 \mathrm{~min}$ at $4{ }^{\circ} \mathrm{C}$. After rinsing with PBS/BSA $1 \%$, cells were incubated with primary antibodies for $60 \mathrm{~min}$ at room temperature. Antibodies used in the different samples were: anti-CD44 (1:200; Abcam), anti-CD90 (1:100; Abcam), anti-CD105 (1:100; Abcam), anti-CD14 (1:200; Abcam), anti-CD34 (1:200; Novus) or anti-CD45 (1:200; Abcam). Finally, cells were washed with PBS/1 \% BSA and incubated for $60 \mathrm{~min}$ at room temperature in the dark with secondary antibodies (goat anti-mouse or goat anti-rabbit) conjugated with fluorescein (FITC; 1:200; Abcam). Samples were analyzed using a Coulter Epics Elite ESP flow cytometer. A minimum of 10,000 forward and side scatter gated events were collected per specimen. Samples were excited at $\lambda=488 \mathrm{~nm}$ and fluorescence was monitored at $\lambda=525 \mathrm{~nm}$. Fluorescence was detected using logarithmic amplification. Mean fluorescence intensity (MFI) values were calculated and recorded automatically.

\section{MTT assay}

The rate of cell proliferation in each sample was evaluated by MTT assay after the third passage. Briefly, about 8,000 cells were seeded in flat-bottomed $200 \mu 1$ microplates, and incubated at $37^{\circ} \mathrm{C}$ in a humidified atmosphere containing $5 \% \mathrm{CO}_{2}$. Quantitative estimates were carried out at 24 and $96 \mathrm{~h}$ of culture. At each time point, $20 \mu \mathrm{l}$ of $0.5 \%$ MTT in PBS was added and, after $3 \mathrm{~h}$ of incubation, the supernatant was removed and replaced with $100 \mu \mathrm{l}$ of DMSO. The optical density of each sample was measured using a microplate spectrophotometer (Titertek Multiskan; DAS, Rome, Italy) at $\lambda=550 \mathrm{~nm}$. Each sample was tested in quadruplicate $(n=4)$.

\section{Statistical analysis}

Differences in cell counts of culture samples 
have been evaluated using two-way ANOVA. Tukey's 'Honest Significant Difference' method has been used as post hoc test.

\section{Results}

\section{Tissue analysis}

Microscopic analysis of tissue from the three samples showed marked differences (Fig. 1). Mature adipocytes were clearly better preserved in the microfat sample, in which the adipose tissue appeared homogeneously spread. In the other two histological preparations, the tissue was more dispersedly scattered and pictures were taken from sites where adipocytes were more densely present; in these preparations, a marked damage is visible at cellular level, although with no evident differences between them.
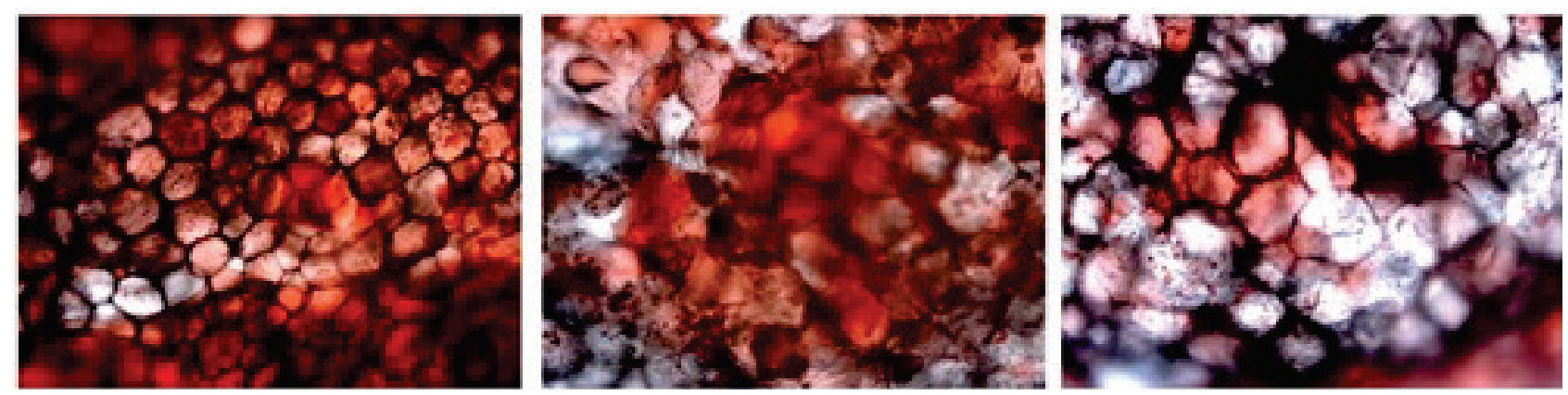

Fig. 1. Photomicrographs of three samples of lipoaspirate stained with Oil Red O. Left: Microfat lipoaspirate harvested with a $3 \mathrm{~mm}$ cannula featuring multiple sharp side holes (1 mm diameter). Middle and right: Nanofat 2.0 and Nanofat specimens, respectively, as obtained after emulsification procedures. Tissue and adipose cells appear well preserved in microfat, whereas a noticeable tissue damage is visible for Nanofat 2.0 and Nanofat. No appreciable difference is evident between them at microscopic level. Magnification: 10x.

\section{Cell growth}

Adherent cells obtained from of all cultured samples showed a fibroblast-like morphology, typical of MSC phenotype but, although the cell shape was similar in all samples, the density was noticeably different, especially at earlier stages. In fact, notwithstanding the same initial volume of each sample, the number of cells at $24 \mathrm{~h}$ of culture was clearly higher in microfat, relatively lower in nanofat 2.0 and quite poor in nanofat culture (Fig. 2A). The same trend was observed at day 4 . At day 8, whereas the cellular density in nanofat remained significantly lower, in nanofat 2.0 it became quite similar to microfat. The higher growth rate of nanofat 2.0 vs. nanofat is clearly visible in Figures $2 \mathrm{~B}-\mathrm{C}$. Notably, from day 4 to day 8 , cell growth rate of nanofat 2.0 appears even greater than microfat. By the enriching strategy described in methods, at the end of the third passage, also in nanofat sample an adequate cell density was achieved. This stage was normally reached at about 16-20 days from liposuction.

\section{Determination of MSC markers}

After the third passage, immunocytochemical staining and flow cytometry were performed. Among the markers investigated, cells showed positive immunostaining for CD44, CD90 and CD105 (Fig. 3A), which are considered specific markers for MSCs (Orbay et al. 2012, Zuk et al. 2002). Consistently, no appreciable immunostaining was observed for CD14, CD34, CD45 (Fig. 3B), typical hematopoietic stem cell markers (Baglioni et al. 2009).

Results from flow cytometry corroborate immunocytochemical observations (Table 1). In fact, high percentages of positive cells (Fig. 3C) were observed for CD44 (96-99\%), for CD90 (65-70\%) and for CD105 (75-82\%). Conversely, very low percentages of positive cells (Fig. 3D) were obtained for CD14 (4-7 \%), for CD34 (7-20\%) and for CD45 (4-13\%).

Table 1. Immunocytochemical phenotype of stem cells from microfat, nanofat 2.0 and nanofat samples, as revealed by flow cytometry. Data were gathered from four patients.

\begin{tabular}{lccc}
\hline \multirow{2}{*}{ Markers } & \multicolumn{3}{c}{ Positive cells (\%) } \\
& Microfat & Nanofat 2.0 & Nanofat \\
\hline CD44 & $98.39 \pm 1.48$ & $98.88 \pm 0.71$ & $96.32 \pm 1.32$ \\
CD90 & $70.00 \pm 2.71$ & $65.58 \pm 2.95$ & $67.38 \pm 2.45$ \\
CD105 & $74.87 \pm 2.58$ & $75.83 \pm 2.88$ & $82.65 \pm 2.07$ \\
CD14 & $3.81 \pm 2.06$ & $5.45 \pm 1.87$ & $6.67 \pm 1.99$ \\
CD34 & $7.84 \pm 2.92$ & $14.86 \pm 2.09$ & $20.01 \pm 1.63$ \\
CD45 & $4.16 \pm 1.95$ & $3.45 \pm 2.72$ & $13.68 \pm 2.09$ \\
\hline
\end{tabular}




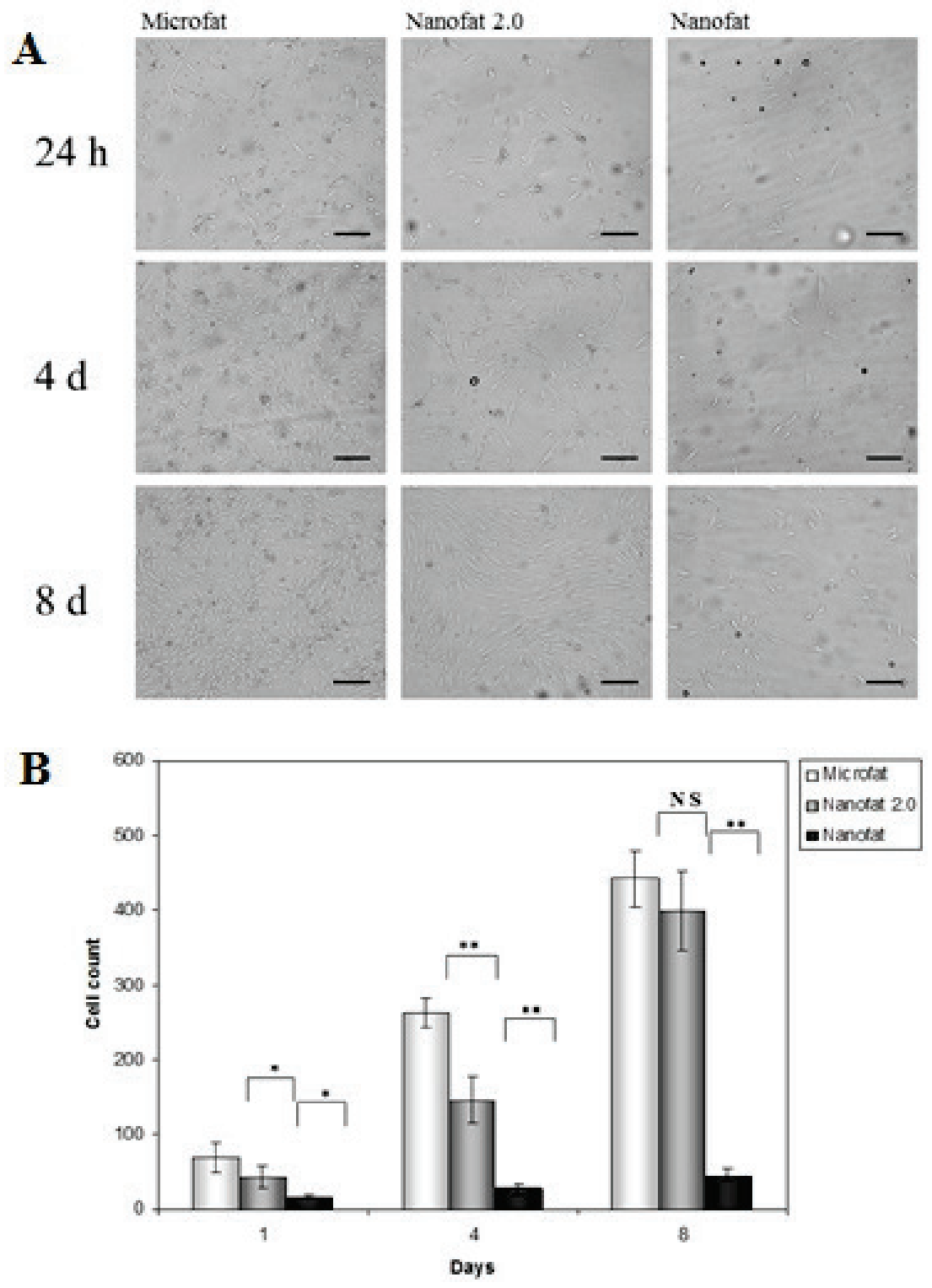

Fig. 2. Cell density and proliferation rate in cultures of microfat, nanofat 2.0 and nanofat samples at first passage, after $24 \mathrm{~h}, 4$ days and 8 days from liposuction. (A) At $24 \mathrm{~h}$, the number of cells is visibly higher in microfat and much lower in nanofat; an intermediate density can be observed in nanofat 2.0. After 4 days, the number of cells is increased in all samples, especially in microfat and nanofat 2.0. At 8 days, a very high density of cells is reached in microfat and nanofat 2.0 cultures, but it is still poor in nanofat. Magnification 10x, scale bar: $100 \mu \mathrm{m}$. (B) Quantitative data of cell counts at first passage after liposuction in microfat, nanofat 2.0 and nanofat samples. Histograms are obtained by averaging the number of cells detected in 5 microscopic fields in zones of higher density for each patient. (C) Growth rate of cells in the three samples. The high growth rate observed in nanofat 2.0 results in a cell density that after 8 days almost equals the microfat sample. Instead, in nanofat sample, the low cell density observed at $24 \mathrm{~h}$ leads a scarce population even at 8 days, given the growth rate particularly low. Data reported in $\mathrm{B}$ and $\mathrm{C}$ were gathered from four patients.

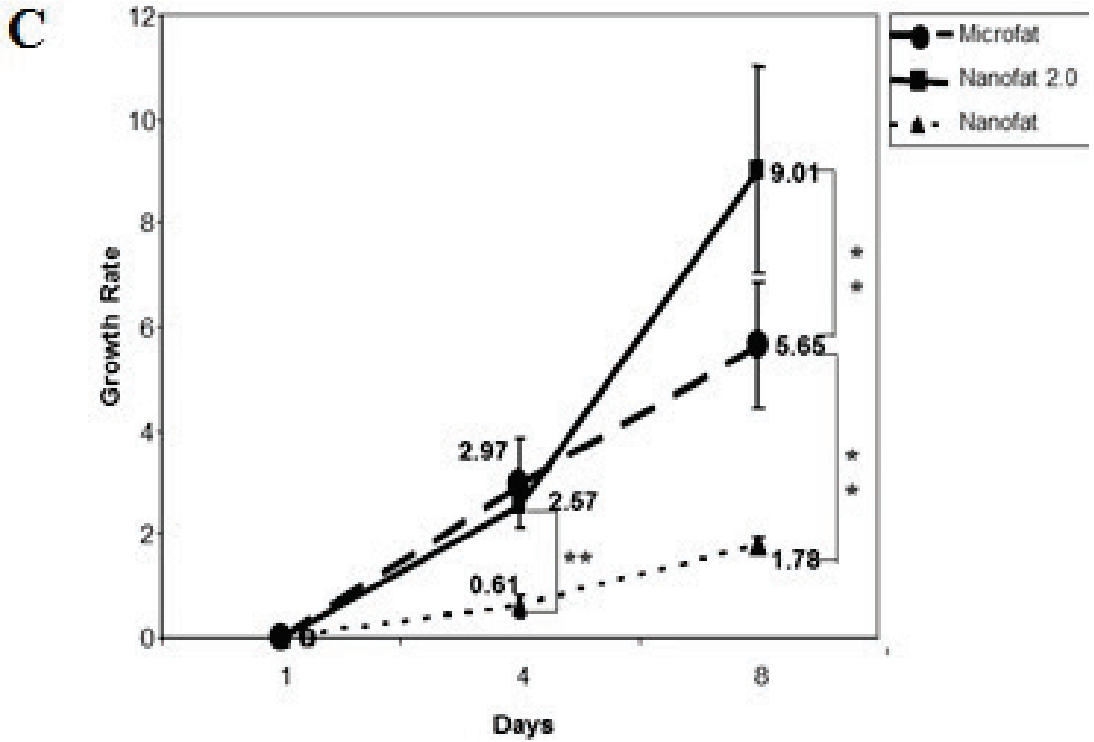




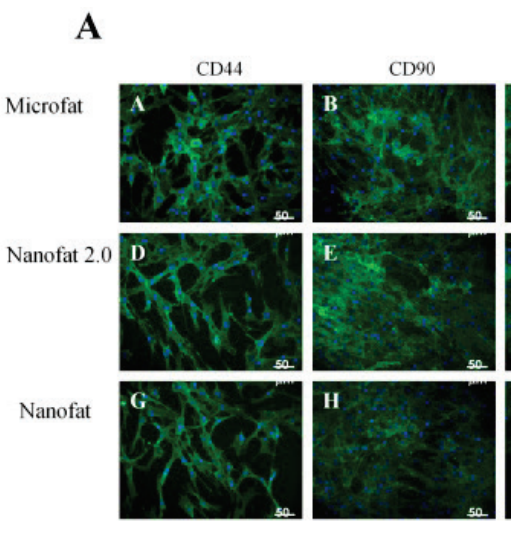

B

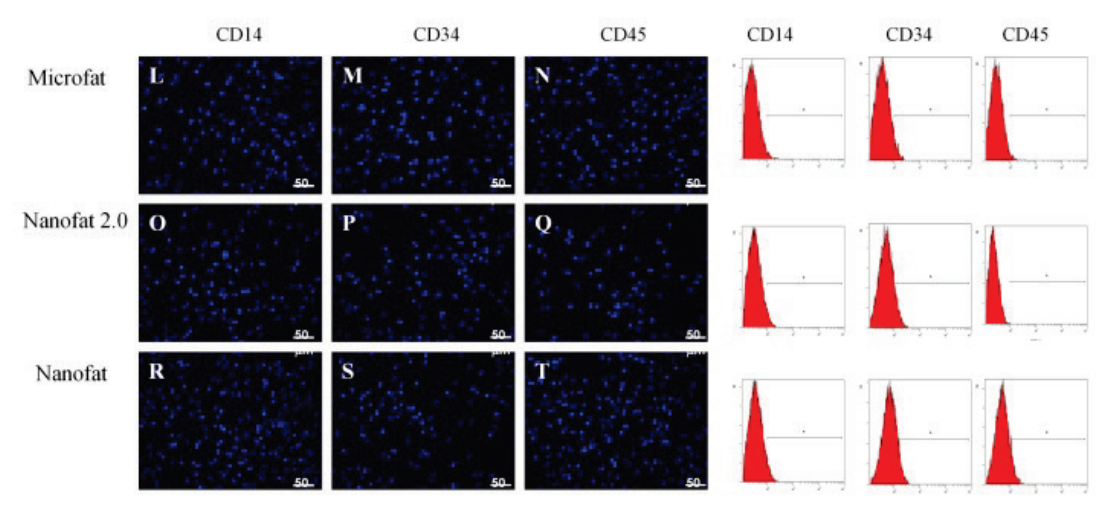

Fig. 3. Immunophenotypical characterizations for markers of mesenchymal stem cells. (A) Photomicrographs at fluorescence microscopy of mesenchymal stem cells from microfat, nanofat 2.0 and nanofat samples. Green fluorescence indicates the positive immunostaining for primary antibodies; blue fluorescence depicts cell nuclei, stained by DAPI. Virtually every cell of all samples is immunopositive for mesenchymal stem cell markers CD44 $(A, D, G), \quad C D 90 \quad(B, E, H), \quad C D 105 \quad(C, F, I)$. (B) No immunostaining is visible for markers considered typical for hematopoietic stem cells CD14 $(\mathrm{L}, \mathrm{O}, \mathrm{R})$ CD34 (M,P,S) and CD45 $(\mathrm{N}, \mathrm{Q}, \mathrm{T})$. The presence of numerous immunonegative cells is documented by DAPI nuclear fluorescence. Magnification: 20x; scale bar: $50 \mu \mathrm{m}$. (C) Histograms showing flow cytometry findings for surface markers in culture of microfat, nanofat 2.0 and nanofat samples. High percentages of positive cells were obtained in all samples for CD44 (96-99\%), CD90 (65-70\%) and CD105 $(75-82 \%)$. On the contrary, very low percentages (D) of positive cells were observed for hematopoietic stem cell markers CD14 (4-7\%), CD34 (7-20\%) and CD45 (4-13\%).
MTT assay

The rate of ASCs proliferation was tested after the third passage. The same amount of stem cells seeded for each sample (about 8,000 cells) led to an even distribution of them, as verified at $24 \mathrm{~h}$ (Fig. 4A). Data from a patient are reported in Figure 4B: at $96 \mathrm{~h}$ a very rapid proliferation was observed for cells of each sample, leading to an increase of the cell number of about four times as much. No evident differences were observed among cells of the three samples, either from a qualitative or quantitative point of view. Similar results were obtained from all patients.

\section{Discussion}

Results obtained show that ASCs can be isolated from each sample investigated (microfat, nanofat and nanofat 2.0), although with a different yield.

Histological inspection at the light microscope after liposuction confirmed the best preservation of mature adipocytes in microfat lipoaspirate, whereas in the other two samples the tissue integrity was highly impaired. However, because of the less mechanical stress of nanofat 2.0 vs. nanofat, it is likely to assume that nanofat 2.0 emulsion still contains a larger amount of
ASCs, growth factors normally present in the adipose tissue, and more numerous cellular debris. Indeed, the presence of adipocyte cellular debris could positively stimulate a mild inflammatory response in the receiving site, with consequent vasodilation, induction of angiogenesis, macrophages recruitment (Janis and Harrison 2014, Sezgin et al. 2014). As a consequence, more cytokines and growth factors would be released, helping stem cells differentiation (Mahadavian Dalavary et al. 2011).

As widely accepted in literature, the MSC nature of cultured cells in this study was assessed by their positive immunoreactivity to specific markers such as CD44, CD90 and CD105 (Baglioni et al. 2009, Orbay et al. 2012, Zuk et al. 2002). They were instead immunonegative to CD34, in apparent contrast with results by Tonnard et al. (2013). These authors indeed considered as ASCs a CD34 positive population. However, as concluded by Gimble and Guilak (2003), such discrepancies might be simply due to differences in cell isolation methods, as well as to the different time of detection. In fact, many studies indicate that ASCs show CD34 antigen only during the first two or three cycles of proliferation (about 10 days), while this marker is permanently expressed in stem cells of hematopoietic 
A

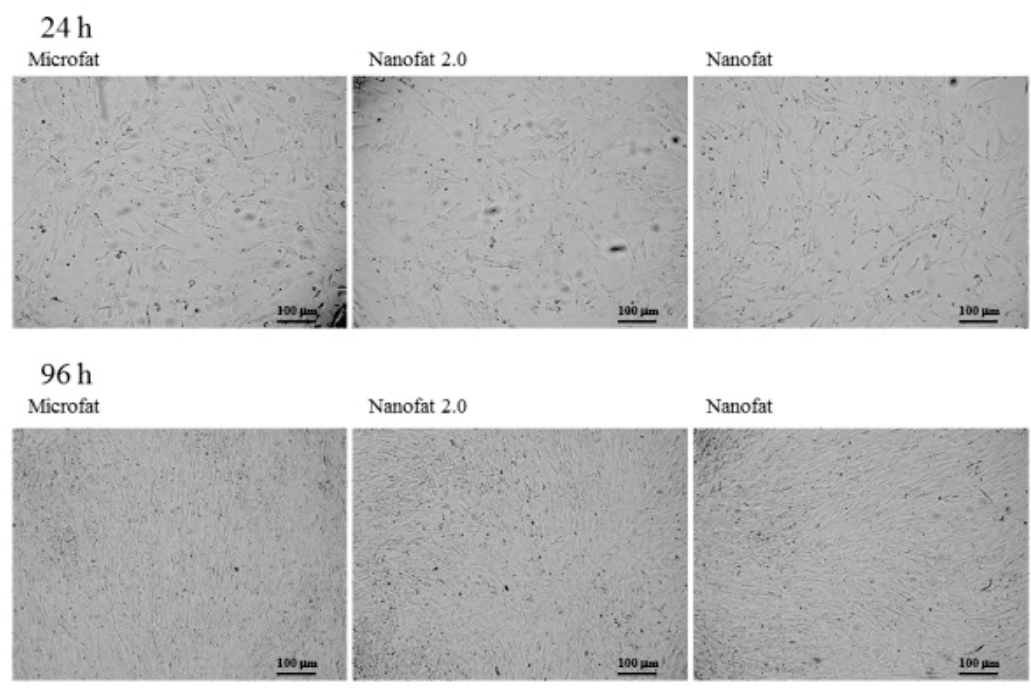

B

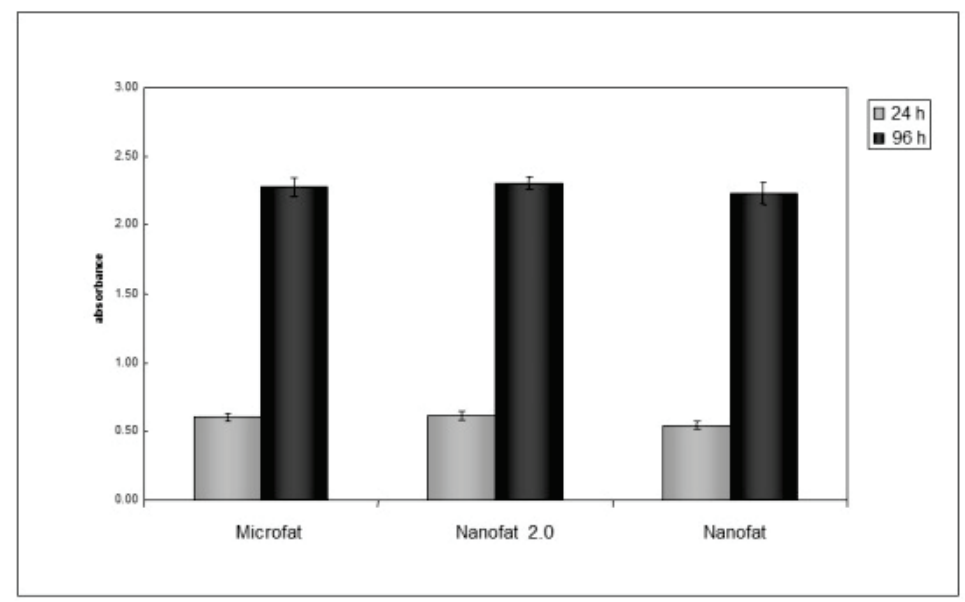

Fig. 4. Cell proliferation tested in the three samples (microfat, nanofat 2.0 and nanofat) after the third passage. For each sample about 8,000 stem cells are seeded and their proliferation was evaluated after 24 and $96 \mathrm{~h}$. (A) The homogenous distribution at $24 \mathrm{~h}$ leads to a confluence pattern at $96 \mathrm{~h}$. (B) Histograms obtained by MTT assay show a number increase of about four times as much. No evident difference is noticeable among cells obtained from the three samples. At each time, data are averaged from values obtained in quadruplicate and presented as mean $\pm \mathrm{SD}$. origin (Mitchell et al. 2006). In the present work, since marker expression was carried out at about 20 days after liposuction, the immunonegativity of ASCs to CD34 is to be considered normal. Besides, they were immunonegative for other hematopoietic markers, such as CD14 and CD45.

In principle, one can conclude that any of the three samples, although with different features, might be suitable for implantation immediately after the harvest. Microfat lipoaspirate would be more advantageous because of its higher content in stem cells; but, according to limitations described in literature, they do not seem able to express entirely their potential. Good chances of stem cell engraftment of nanofat may account for appreciable clinical results obtained using nanofat (Tonnard et al. 2013, Tamburino et al. 2016); yet, the technique implies the yield of a modest amount of stem cells, showing a low proliferation rate. Therefore, nanofat 2.0 might represent an excellent compromise because it includes positive aspects of the other two samples, but avoiding some unfavorable side effects. In fact, common complications of microfat grafting, such as an unpredictable loss of its volume, risk of undercorrection or overcorrection, contour irregularities and cysts of liponecrosis (Kim et al. 2016), are not described in nanofat grafting.

Beside, nanofat may be injected in an intradermal plane through very small needles of $27 \mathrm{G}$, without the need of cannulas. More notably, when compared with nanofat, nanofat 2.0 might be able to induce faster and more effective regenerative effects, because of the higher density of stem cells, featuring a higher proliferative rate, especially in the first few days after the harvest.

Of course, the stage at which this characterization was carried out is not compatible with the use for clinical purposes, since the procedure of ASCs expansion clearly exceeds the minimal manipulations 
widely required for human trials. However, results obtained at $24 \mathrm{~h}$ provide useful indications about the density and viability of stem cells already present at the time of the liposuction. Thus, nanofat 2.0 may represent a valuable version of fat grafting for improving skin quality or wound healing, especially when large volume augmentation is not required.

According to newer concepts of fat grafting, nanofat 2.0 could be considered stem cells transplantation rather than a simple adipocytes graft. As also proposed for lipogems (Bianchi et al. 2013), when safe procedures of cryopreservation and storing will be standardized, it might represent a ready-to-use product for successive applications in the same patient or even for allogeneic transplantations, given the low immunogenicity and immunosuppressive properties of ASCs. In this case, fat harvesting and procedures of preparation would be conveniently spared. Moreover, although designed for plastic and aesthetic surgery, it may be useful for several purposes as a SVF-like compound. For example, due to the remarkable multilineage differentiation ability of ASCs, it might be suitable for a variety of treatments, form bone fractures to peripheral nerve regenerations.

\section{Conflict of Interest}

There is no conflict of interest.

\section{Acknowledgements}

This work was supported by University of Catania, Italy. FIR 2014 - 20722142002.

\section{References}

BAGLIONI S, FRANCALANCI M, SQUECCO R, LOMBARDI A, CANTINI G, ANGELI R, GELMINI S, GUASTI D, BENVENUTI S, ANNUNZIATO F, BANI D, LIOTTA F, FRANCINI F, PERIGLI G, SERIO M, LUCONI M: Characterization of human adult stem-cell populations isolated from visceral and subcutaneous adipose tissue. FASEB J 23: 3494-3505, 2009.

BIANCHI F, MAIOLI M, LEONARDI E, OLIVI E, PASQUINELLI G, VALENTE S, MENDEZ AJ, RICORDI C, RAFFAINI M, TREMOLADA C, VENTURA C: A new nonenzymatic method and device to obtain a fat tissue derivative highly enriched in pericyte-like elements by mild mechanical forces from human lipoaspirates. Cell Transplant 22: 2063-2077, 2013.

BUTALA P, HAZEN A, SZPALSKI C, SULTAN SM, COLEMAN SR, WARREN SM: Endogenous stem cell therapy enhances fat graft survival. Plast Reconstr Surg 130: 293-306, 2012.

CALABRESE G, GIUFFRIDA R, LO FURNO D, PARRINELLO NL, FORTE S, GULINO R, COLAROSSI C, SCHINOCCA LR, GIUFFRIDA R, CARDILE V, MEMEO L: Potential effect of CD271 on human mesenchymal stromal cell proliferation and differentiation. Int J Mol Sci 16: 15609-15624, 2015.

CHARLES-DE-SÁ L, GONTIJO-DE-AMORIM NF, MAEDA TAKIYA C, BOROJEVIC R, BENATI D, BERNARDI P, SBARBATI A, RIGOTTI G: Antiaging treatment of the facial skin by fat graft and adipose-derived stem cells. Plast Reconstr Surg 135: 999-1009, 2015.

GIMBLE J, GUILAK F: Adipose-derived adult stem cells: isolation, characterization, and differentiation potential. Cytotherapy 5: 362-369, 2003.

JANIS JE, HARRISON B: Wound healing: part I. Basic science. Plast Reconstr Surg 133: 199e-207e, 2014.

KALININA N, KHARLAMPIEVA D, LOGUINOVA M, BUTENKO I, POBEGUTS O, EFIMENKO A, AGEEVA L, SHARONOV G, ISCHENKO D, ALEKSEEV D, GRIGORIEVA O, SYSOEVA V, RUBINA K, LAZAREV V, GOVORUN V: Characterization of secretomes provides evidence for adipose-derived mesenchymal stromal cells subtypes. Stem Cell Res Ther 6: 221, 2015.

KIM IA, KELLER G, GROTH MJ, NABILI V: The downside of fat: avoiding and treating complications. Facial Plast Surg 32: 556-559, 2016.

KØLLE SF, FISCHER-NIELSEN A, MATHIASEN AB, ELBERG JJ, OLIVERI RS, GLOVINSKI PV, KASTRUP J, KIRCHHOFF M, RASMUSSEN BS, TALMAN ML, THOMSEN C, DICKMEISS E, DRZEWIECKI KT: Enrichment of autologous fat grafts with ex-vivo expanded adipose tissue-derived stem cells for graft survival: a randomised placebo-controlled trial. Lancet 382: 1113-1120, 2013. 
LO FURNO D, PELLITTERI R, GRAZIANO AC, GIUFFRIDA R, VANCHERI C, GILI E, CARDILE V: Differentiation of human adipose stem cells into neural phenotype by neuroblastoma- or olfactory ensheathing cells-conditioned medium. J Cell Physiol 228: 2109-2118, 2013.

MAHDAVIAN DELAVARY B, VAN DER VEER WM, VAN EGMOND M, NIESSEN FB, BEELEN RH: Macrophages in skin injury and repair. Immunobiology 216: 753-762, 2011.

MATSUMOTO D, SATO K, GONDA K, TAKAKI Y, SHIGEURA T, SATO T, AIBA-KOJIMA E, IIZUKA F, INOUE K, SUGA H, YOSHIMURA K: Cell-assisted lipotransfer: supportive use of human adipose-derived cells for soft tissue augmentation with lipoinjection. Tissue Eng 12: 3375-3382, 2006.

MITCHELL J, MCINTOSH K, ZVONIC S, GARRETT S, FLOYD ZE, KLOSTER A, DI HALVORSEN Y, STORMS RW, GOH B, KILROY G, WU X, GIMBLE JM: Immunophenotype of human adipose-derived cells: temporal changes in stromal-associated and stem cell-associated markers. Stem Cells 24: 376-385, 2006.

ORBAY H, TOBITA M, MIZUNO H: Mesenchymal stem cells isolated from adipose and other tissues: basic biological properties and clinical applications. Stem Cells Int 2012: 461718, 2012.

SEZGIN B, OZMEN S, BULAM H, OMEROGLU S, YUKSEL S, CAYCI B, PEKER T: Improving fat graft survival through preconditioning of the recipient site with microneedling. J Plast Reconstr Aesthet Surg 67: 712-720, 2014.

TAMBURINO S, LOMBARDO GA, TARICO MS, PERROTTA RE: The role of nanofat grafting in vulvar lichen sclerosus: a preliminary report. Arch Plast Surg 43: 93-95, 2016.

TONNARD P, VERPAELE A, PEETERS G, HAMDI M, CORNELISSEN M, DECLERCQ H: Nanofat grafting: basic research and clinical applications. Plast Reconstr Surg 132: 1017-1026, 2013.

ULLAH I, SUBBARAO RB, RHO GJ: Human mesenchymal stem cells - current trends and future prospective. Biosci Rep 35: e00191, 2015.

YOSHIMURA K, SATO K, AOI N, KURITA M, HIROHI T, HARII K: Cell-assisted lipotransfer for cosmetic breast augmentation: supportive use of adipose-derived stem/stromal cells. Aesthetic Plast Surg 32: 48-55, 2008.

ZUK PA, ZHU M, ASHJIAN P, DE UGARTE DA, HUANG JI, MIZUNO H, ALFONSO ZC, FRASER JK, BENHAIM P, HEDRICK MH: Human adipose tissue is a source of multipotenti stem cells. Mol Biol Cell 13: 4279-4295, 2002. 